\title{
Statistical Estimation of Atomic Positions from Exit Wave Reconstruction with a Precision in the Picometer Range
}

\author{
Sara Bals, ${ }^{1, *}$ Sandra Van Aert, ${ }^{1}$ Gustaaf Van Tendeloo, ${ }^{1}$ and David Ávila-Brande ${ }^{2}$ \\ ${ }^{1}$ Electron Microscopy for Materials Science (EMAT), University of Antwerp, Groenenborgerlaan 171, 2020 Antwerp, Belgium \\ ${ }^{2}$ Departamento Química Inorgánica, Facultad CC. Químicas, Universidad Complutense E-28040, Madrid, Spain
}

(Received 15 November 2005; published 10 March 2006)

\begin{abstract}
The local structure of $\mathrm{Bi}_{4} \mathrm{~W}_{2 / 3} \mathrm{Mn}_{1 / 3} \mathrm{O}_{8} \mathrm{Cl}$ is determined using quantitative transmission electron microscopy. The electron exit wave, which is closely related to the projected crystal potential, is reconstructed and used as a starting point for statistical parameter estimation. This method allows us to refine all atomic positions on a local scale, including those of the light atoms, with a precision in the picometer range. Using this method one is no longer restricted to the information limit of the electron microscope. Our results are in good agreement with x-ray powder diffraction data demonstrating the reliability of the method. Moreover, it will be shown that local effects can be interpreted using this approach.
\end{abstract}

DOI: 10.1103/PhysRevLett.96.096106

PACS numbers: 68.37.Lp, 42.30.Wb, 43.50.+y

A new phase with nominal composition $\mathrm{Bi}_{4} \mathrm{~W}_{2 / 3} \mathrm{Mn}_{1 / 3} \mathrm{O}_{8} \mathrm{Cl}$ has recently been synthesized [1]. The interest in this compound, which is based on a Sillén-Aurivillius intergrowth [2], is found in the resemblance of the structure with, e.g., the high $T_{c}$ superconducting bismuth phases $[3,4]$ and the manganese based CMR materials $[5,6]$.

One of the standard techniques to recover the atomic structure of new compounds is x-ray (powder) diffraction. Although this technique yields a very good precision, the results are only obtained on an average scale. This is a disadvantage since slight deviations from the average structure, even on an atomic scale, can have great influence on the physical properties of the compound being investigated. To study the atomic structure on a local scale, transmission electron microscopy (TEM) offers better perspectives. However, interpretation of conventional high resolution TEM (HRTEM) images is far from intuitive and image simulations of a tentative model are required. Furthermore, the HRTEM images are often very insensitive to the positions of light atomic columns.

Recently, exit wave reconstruction in TEM has been applied to determine the positions of the atomic columns, including those of light atomic columns such as oxygen $[7,8]$. The reconstruction requires a focal series of 10-20 images. The idea is to invert the image formation process so that lens aberrations can be eliminated and all information up to the microscope's information limit $\left(\rho_{I}\right)$ is resolved [9]. This can be done directly with a minimal amount of prior knowledge. In this way, the phase and the amplitude of the exit wave (which is in fact the complex electron wave when leaving the specimen) are extracted, whereas a conventional TEM image only yields the image intensity. The spatial information which can be visually extracted from such an exit wave reconstruction is still restricted to $\rho_{I}$, the information limit of the microscope, which in the best case is of the order of $1 \AA$.
In this study, we have used the exit wave as a starting point for quantitative refinement of the atom positions using statistical parameter estimation theory [10]. This approach allows one to determine local interatomic distances in a quantitative manner without being restricted by the information limit of the microscope. Alternatively, the exit wave can also be reconstructed using off-axis holography [11] and can be used as a starting point for further refinement as well. Here, we will first demonstrate that results obtained with a precision in the picometer range using the statistical parameter estimation theory are comparable to the $\mathrm{x}$-ray diffraction data acquired for the $\mathrm{Bi}_{4} \mathrm{~W}_{2 / 3} \mathrm{Mn}_{1 / 3} \mathrm{O}_{8} \mathrm{Cl}$ compound [1]. Next, we will show that local deviations in the interatomic distances can be detected and quantified using this method.

A polycrystalline sample with nominal composition $\mathrm{Bi}_{4} \mathrm{~W}_{2 / 3} \mathrm{Mn}_{1 / 3} \mathrm{O}_{8} \mathrm{Cl}$ is prepared by heating stoichiometric amounts of the oxides $\mathrm{Bi}_{2} \mathrm{O}_{3}, \mathrm{WO}_{3}, \mathrm{Mn}_{2} \mathrm{O}_{3}$, and $\mathrm{BiOCl}$ at $993 \mathrm{~K}$ for a week in an evacuated $(P=$ $10^{-4}$ torr) sealed silica tube. The sample is then slowly cooled to room temperature during $24 \mathrm{~h}$. Samples for TEM are prepared by ultrasonic dispersion of the crystals in $n$-butanol. Drops of this dispersion are deposited on a holey carbon-coated copper grid. From conventional TEM techniques combined with $\mathrm{x}$-ray diffraction, the lattice parameters $(a=5.467 \AA, b=5.467 \AA, c=14.159 \AA)$ and space group $(\mathrm{Cm} 2 \mathrm{~m})$ of the $\mathrm{Bi}_{4} \mathrm{~W}_{2 / 3} \mathrm{Mn}_{1 / 3} \mathrm{O}_{8} \mathrm{Cl}$ compound have been determined [1].

For exit wave reconstruction, a Philips CM30 with an information limit of $1.1 \AA$ and equipped with a slow-scan CCD camera is used. Prior to acquisition, an amorphous area of the specimen is used to determine the starting defocus as accurately as possible and to minimize coma, twofold, and threefold astigmatism. A series of 20 images is recorded from the same area (imaged along the [1 10$]$ zone axis) with a starting defocus of $-140 \mathrm{~nm}$ and an 
equidistant focal decrease of $2 \mathrm{~nm}$. The actual reconstruction of the exit wave is carried out using the TrueImage software [12]. After reconstruction the residual aberrations are corrected using the standard techniques in TrueImage and the complex exit wave is split into a phase and amplitude image. The result of this procedure is shown in Figs. 1(a) and 1(b). The phase of the exit wave is proportional to the projected electrostatic potential of the structure. Based on the positions of the atoms in the parent Sillén-Aurivillius phase [1] we can therefore directly indicate the different atomic columns present in $\mathrm{Bi}_{4} \mathrm{Mn}_{1 / 3} \mathrm{~W}_{2 / 3} \mathrm{O}_{8} \mathrm{Cl}$. An enlarged section of the phase image is presented in Fig. 1(c), where an overlay indicates the positions of the different types of atomic columns. It should be noted that also the positions of the light atomic columns are imaged. Indeed, the phase image clearly shows the position of the $\mathrm{Cl}$ atomic columns as well as the projected $\left\{\mathrm{WO}_{6}\right\} /\left\{\mathrm{MnO}_{6}\right\}$ octahedra, with the fainter dots corresponding to the $\mathrm{O}(1)$ and $\mathrm{O}(2)$ atomic columns. Adjacent to the $\mathrm{Bi}(2)$ columns, smaller dots are observed, this time corresponding to the $\mathrm{O}(3)$ atomic columns. Around the $\mathrm{Bi}(1)$ columns the closest $\mathrm{O}(3)$ atomic columns are not detected separately, but an elongation of the Bi dots is observed. Next, we will show that using statistical parameter estimation we are able to determine the projected interatomic distance between these columns, even though

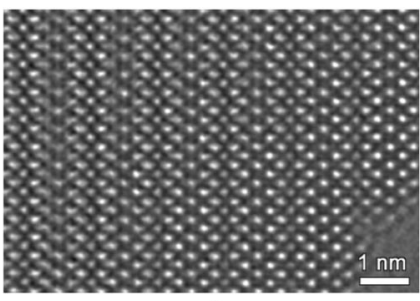

a)

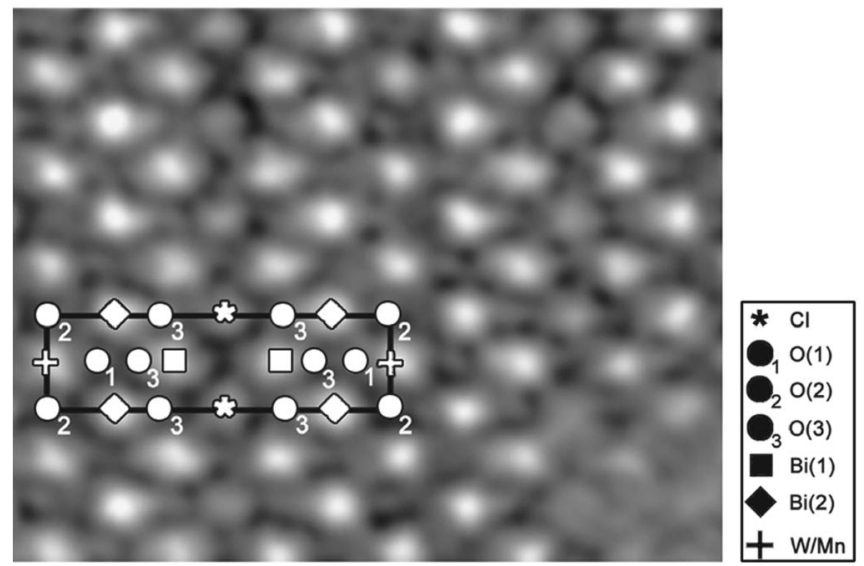

c)

FIG. 1. (a) Phase image and (b) amplitude image of the reconstructed exit wave. An enlarged section of the phase image is presented in (c) where the projected unit cell is outlined. It should be noted that the position of the $\mathrm{W} / \mathrm{Mn}$ column overlaps with another $\mathrm{O}(2)$ atomic column. this distance is beyond the information limit of the microscope.

Although the use of exit wave reconstruction has several advantages, such as the ability to interpret the phase image without the need for image simulations, the reconstructed exit wave does not directly provide quantitative numbers for the atomic positions. Therefore, we have used the phase of the exit wave as an input for further refinement using statistical parameter estimation. In this manner, it is possible to determine the structure with a precision that is orders of magnitude better than the information limit of the electron microscope. This, however, requires a quantitative, model-based method, in which the phase of the reconstructed exit wave is considered as a data plane from which the unknown structure parameters, such as the atomic column positions, have to be estimated in a statistical way. A comprehensive report of this model-based method can be found in $[10,13]$. The key to successful application is the availability of a parametric model describing the expectations of the pixel values in the reconstructed phase. Nowadays, the physics behind the electronobject interaction is sufficiently well understood to have an expression describing the phase of an electron exit wave. It is known that thin specimens act as pure phase objects of which the phase of the exit wave can be modeled as a superposition of Gaussian peaks [14]. The expectations at the pixel $(k, l)$ at the position $\left(x_{k} y_{l}\right)$ are given by the following function:

$f_{k l}(\theta)=\zeta+\sum_{i=1}^{I} \sum_{m_{i}=1}^{M_{i}} \alpha_{i} \exp \left(\frac{-\left(x_{k}-\beta_{x_{m_{i}}}\right)^{2}-\left(y_{l}-\beta_{y_{m_{i}}}\right)^{2}}{2 \rho_{i}^{2}}\right)$

where $\zeta$ is a constant background, $\alpha_{i}$ and $\rho_{i}$ are the column type dependent height and width of the Gaussian peak, respectively, $\beta_{x_{m_{i}}}$ and $\beta_{y_{m_{i}}}$ are the $x$ and $y$ coordinate of the $m_{i}$ th atom column, respectively. In this expression, the index $i$ refers to a particular column type, e.g., W/Mn or $\mathrm{Cl}$, and the index $m_{i}$ refers to the $m$ th column of column type $i$. The unknown parameters of the model, that is, $\theta=$ $\left(\beta_{x_{1}} \ldots \beta_{x_{M_{I}}} \beta_{y_{1_{1}}} \ldots \beta_{y_{M_{I}}} \alpha_{1} \ldots \alpha_{I} \rho_{1} \ldots \rho_{I} \zeta\right)^{T}$, are estimated in the least squares sense. The uniformly weighted least squares estimates $\hat{\theta}$ are given by the values of $t$ that minimize the uniformly weighted least squares criterion:

$$
\hat{\theta}=\arg \min _{t} \sum_{k=1}^{K} \sum_{l=1}^{L}\left[w_{k l}-f_{k l}(t)\right]^{2},
$$

with $w_{k l}$ the value of the reconstructed phase at the pixel $(k, l)$. Note that for pixel values which are independent and identically normally distributed about the expectations, the uniformly weighted least squares estimator is identical to the maximum likelihood estimator, which is known to have optimal statistical properties [10]. As shown in [15], this assumption about the statistical distribution of the pixel values is a reasonable one. 
Uniformly weighted least squares estimation has been applied to the experimental phase shown in Fig. 2(a) using the model given by Eq. (1). Note that this estimation procedure, that is, finding the global minimum of the uniformly weighted least squares criterion, is an iterative numerical procedure. Since the number of unknown parameters is high, it is quite possible that this minimization ends up at a local minimum instead of at the global minimum. In order to avoid this, good starting values for the parameters are required. In other words, the structure has to be resolved. This corresponds to $\mathrm{x}$-ray crystallography, where one first has to resolve the structure and afterwards one has to refine the structure. Resolving the structure is here done by means of exit wave reconstruction. Figures 2(b) and 2(c) show the model evaluated at the estimated parameters $\hat{\theta}$, that is, $f_{k l}(\hat{\theta})$. In a sense, these figures can be regarded as optimal reconstructions of the phase of the exit wave. From the estimated atomic column position coordinates $\hat{\beta}_{x_{m_{i}}}$ and $\hat{\beta}_{y_{m_{i}}}$, interatomic distances, as indicated in Fig. 3 , have been computed using the invariance property of the maximum likelihood estimator [16]. From sets of equivalent distances, mean interatomic distances and their corresponding standard deviations have been calculated. The results are presented in Table I. It can be seen that the standard deviation, being a measure of the precision, is in the picometer range. In order to judge the adequateness of the model used, the so-called residual plot, normal probability plot, and quantitative test for zero mean of the residuals have been performed $[10,13]$. In addition, the reliability of the method is confirmed by comparing the

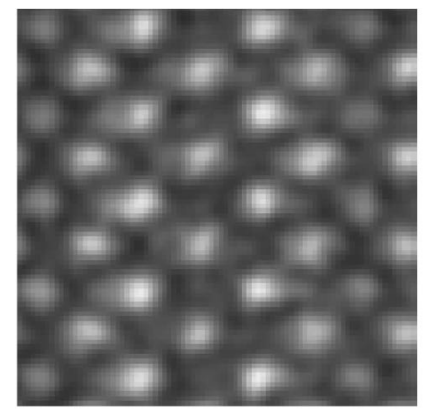

a)

\begin{tabular}{|l|}
\hline $\mathrm{Cl}$ \\
$\square \mathrm{Bi}(1)$ \\
$\diamond \mathrm{Bi}(2)$ \\
$+\mathrm{W} / \mathrm{Mn}$ \\
$\circ \mathrm{O}(1), \mathrm{O}(2), \mathrm{O}(3)$ \\
\hline
\end{tabular}

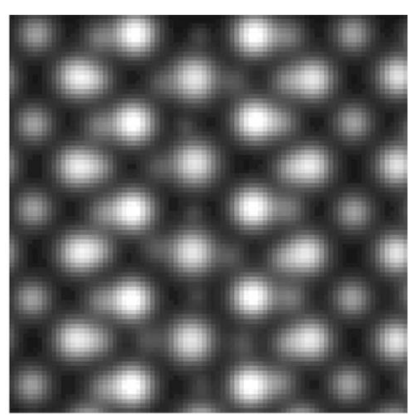

b)

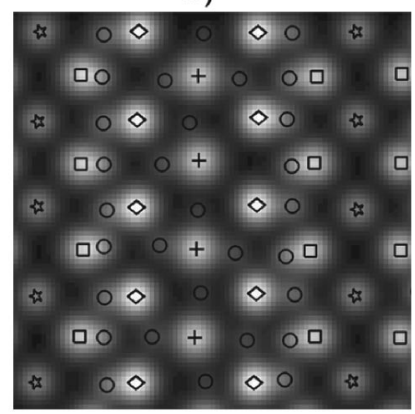

c)
FIG. 2. (a) Experimental phase image and (b) model for the phase evaluated at the estimated parameters. An overlay indicating the different types of atomic columns is shown in (c). computed interatomic distances to the corresponding distances as obtained from Rietveld refinement [1].

As can be seen from the projected interatomic distances in Table I, a good agreement is found when comparing $\mathrm{x}$ ray diffraction and exit wave reconstruction combined with statistical parameter estimation. Moreover, using our method, projected interatomic distances can be determined with a precision in the picometer range, which is quite remarkable when using (quantitative) TEM. Alternatively, quantitative electron diffraction techniques such as parallel recording of dark-field images (PARODI) [17] allow to reach picometer accuracy when measuring lattice displacements by comparing experimental patterns with simulations. However, a major advantage of our (real space) procedure compared to electron and x-ray diffraction is that it results in numbers for the atomic positions determined on a more local scale. This is important since local distortions or other deviations from the perfect (or average) structure can have great influence on the physical properties of solid state materials. Therefore, statistical parameter estimation is a very promising approach when studying and optimizing the exact atomic arrangement at, e.g., interface structures, grain boundaries, new compounds, etc.

One of the local scale effects is described next: the standard deviation obtained for the projected interatomic distance $d_{3}$ between the $\mathrm{W} / \mathrm{Mn}$ atomic columns and the $\mathrm{O}$ columns at the apical positions $[\mathrm{O}(1)]$ is a factor of 2 larger compared to the standard deviation of the projected interatomic distances $d_{1}$ and $d_{2}$ between the $\mathrm{O}$ columns at the equatorial positions $[\mathrm{O}(2)]$ and the $\mathrm{W} / \mathrm{Mn}$ columns. However, if only statistical fluctuations of the pixel values contribute to the standard deviation, one would expect these standard deviations to have approximately the same value [18]. Therefore, there must be a further dominant effect which contributes to the standard deviation of the $\mathrm{W} / \mathrm{Mn}-\mathrm{O}(1)$ distances. This effect can be understood in terms of the Jahn-Teller distortion. Part of the W cations along the columns is replaced by $\mathrm{Mn}$ cations and it is known that $\mathrm{Mn}^{3+}$ cations cause a Jahn-Teller effect (compression or elongation) of the $\mathrm{O}$ octahedra surrounding $\mathrm{Mn}^{3+}$ in order to break down the degeneration of their

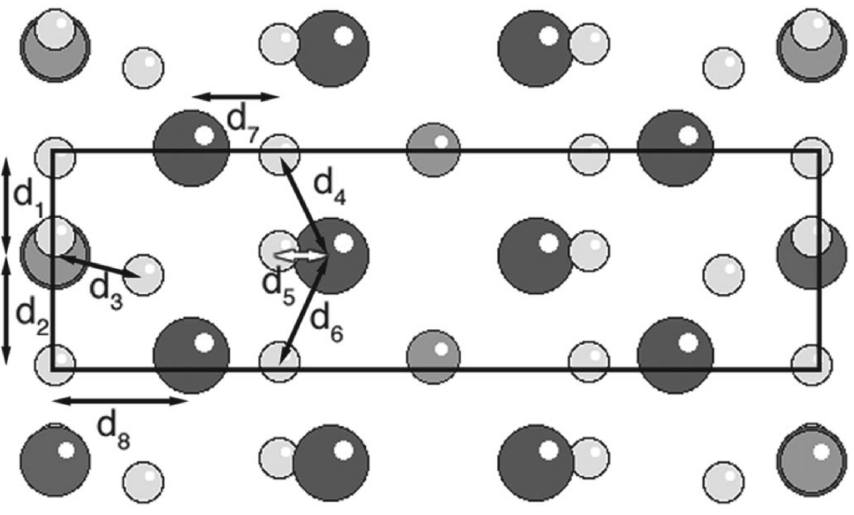

FIG. 3. Projected atomic structure where the interatomic distances that are listed in Table I are indicated. 
TABLE I. Projected interatomic distances obtained from x-ray diffraction and exit wave reconstruction combined with statistical parameter estimation

\begin{tabular}{ccc}
\hline \hline $\begin{array}{c}\text { Mean interatomic } \\
\text { distance }(\AA)\end{array}$ & $\begin{array}{c}\text { Phase of reconstructed } \\
\text { exit wave }\end{array}$ & $\begin{array}{c}\text { X-ray } \\
\text { diffraction }\end{array}$ \\
\hline$d_{1}$ & $1.83 \pm 0.05$ & $1.79 \pm 0.02$ \\
$d_{2}$ & $2.08 \pm 0.06$ & $2.07 \pm 0.02$ \\
$d_{3}$ & $1.8 \pm 0.1$ & $1.70 \pm 0.02$ \\
$d_{4}$ & $2.10 \pm 0.03$ & $2.18 \pm 0.05$ \\
$d_{5}$ & $1.04 \pm 0.04$ & $0.96 \pm 0.07$ \\
$d_{6}$ & $2.29 \pm 0.05$ & $2.14 \pm 0.05$ \\
$d_{7}$ & $1.43 \pm 0.05$ & $1.63 \pm 0.03$ \\
$d_{8}$ & $2.6 \pm 0.1$ & $2.57 \pm 0.03$ \\
\hline \hline
\end{tabular}

energy levels [19]. Since magnetic susceptibility measurements for $\mathrm{Bi}_{4} \mathrm{Mn}_{1 / 3} \mathrm{~W}_{2 / 3} \mathrm{O}_{8} \mathrm{Cl}$ indeed indicate $\mathrm{Mn}^{3+}$ [1], we can thus expect a Jahn-Teller distortion to be present in this case. In Table I, it can be seen that the projected distance $d_{3}$ from the $\mathrm{W} / \mathrm{Mn}$ columns to the $\mathrm{O}$ columns at the apical position is smaller compared to the projected distances $d_{1}$ and $d_{2}$ between the $\mathrm{W} / \mathrm{Mn}$ columns and the $\mathrm{O}$ columns at the equatorial position. We can therefore conclude that the Jahn-Teller distortion will cause a compression of the $\mathrm{O}$ octahedra in this case. This compression does not influence the position of the equatorial O columns, but is responsible for a difference in the projected distance between $\mathrm{Mn}^{3+}$ and $\mathrm{O}$ at the apical position and the corresponding distance between $\mathrm{W}$ and $\mathrm{O}$ at the apical position. This variation is responsible for the larger standard deviation found by statistical parameter estimation. Our method clearly indicates a locally varying $\mathrm{W} / \mathrm{Mn}-\mathrm{O}(2)$ distance, whereas $\mathrm{x}$-ray diffraction yields one average distance without any indication that the $\mathrm{W} / \mathrm{Mn}-\mathrm{O}(2)$ distance shows a variation.

Finally, it is found that the projected distance $\left(d_{5}\right)$ between the $\mathrm{Bi}(1)$ column and the adjacent $\mathrm{O}$ column $(d=$ $1.04 \pm 0.04 \AA$ ) is beyond the information limit of the microscope $\left(\rho_{I}=1.1 \AA\right)$, resulting in one elongated blob in the phase image rather than in two separate dots. Nevertheless, this distance can be determined with picometer precision by applying statistical parameter estimation and the knowledge that the object as projected in the phase image actually consists of two atomic columns. This indicates the importance of considering precision rather than resolution.

In conclusion, the combination of statistical parameter estimation and exit wave reconstruction allows one to determine the structure of $\mathrm{Bi}_{4} \mathrm{Mn}_{1 / 3} \mathrm{~W}_{2 / 3} \mathrm{O}_{8} \mathrm{Cl}$ with a minimal amount of prior knowledge on a local scale with a precision in the picometer range. Furthermore, interatomic distances beyond the information limit of the electron microscope can be estimated. After the validity of this approach is confirmed by comparison of our technique with x-ray diffraction, local effects, being Jahn-Teller distortions, are investigated. It is clear that exit wave reconstruction, combined with statistical parameter estimation, is a very powerful technique which can be applied to resolve the (unknown) structure of new and existing compounds.

S. Bals and S. Van Aert gratefully acknowledge the financial support of the Fund for Scientific ResearchFlanders (FWO). The authors would like to thank Professor A. Abakumov for very interesting discussions. This work has been performed within the IAP V project

*Electronic address: Sara.Bals@ua.ac.be

[1] D. Ávila Brande, L. Otero-Díaz, A. Landa-Cánovas, S. Bals, and G. Van Tendeloo, Eur. J. Inorg. Chem. (to be published).

[2] B. Aurivillius, Chem. Scr. 23, 143 (1984).

[3] K. A. Yee, T. A. Albright, D. Jung, and M. H. Whangbo, Angew. Chem. 28, 750 (1989).

[4] R. L. Withers, J. G. Thompson, L. R. Wallenberg, J. D. Fitzgerald, J. S. Anderson, and B. G. Hyde, J. Phys. C 21, 6067 (1988).

[5] C. N. Rao and B. Raveau, Colossal Magnetoresistence, Charge Ordering and Related Properties of Manganese Oxides (World Scientific, Singapore, 1998).

[6] A. P. Ramirez, J. Phys. Condens. Matter 9, 8171 (1997).

[7] C. L. Jia, M. Lentzen, and K. Urban, Science 299, 870 (2003).

[8] J. Ayache, C. Kisielowski, and R. Kilaas, J. Mater. Sci. 40, 3091 (2005).

[9] W. M. J. Coene, A. Thust, M. op de Beeck, and D. Van Dyck, Ultramicroscopy 64, 109 (1996).

[10] A. J. den Dekker, S. Van Aert, A. van den Bos, and D. Van Dyck, Ultramicroscopy 104, 83 (2005).

[11] H. Lichte, Advances in Optical and Electron Microscopy 12, 25 (1991).

[12] Program TrueImage: Focal-Series Reconstruction Package (Version 1.0.2) (FEI Company, Hillsboro, Oregon, 2003).

[13] S. Van Aert, A. J. den Dekker, A. van den Bos, D. Van Dyck, and J.H. Chen, Ultramicroscopy 104, 107 (2005).

[14] D. Van Dyck, Adv. Imaging Electron Phys. 123, 105 (2002).

[15] M.A. O. Miedema, A. van den Bos, and A.H. Buist, IEEE Trans. Instrum. Meas. 43, 181 (1994).

[16] A. M. Mood, F. A. Graybill, and D. C. Boes, Introduction to the Theory of Statistics (McGraw-Hill, New York, 1974), 3rd ed.

[17] L. Wu, Y. Zhu, and J. Tafto, Phys. Rev. Lett. 85, 5126 (2000).

[18] S. Van Aert, A. J. den Dekker, D. Van Dyck, and A. van den Bos, J. Struct. Biol. 138, 21 (2002).

[19] T. Negas and R.S. Roth, J. Solid State Chem. 3, 323 (1971). 